\section{Practical Field Use of Eye-tracking Devices for Consumer Research in the Retail Environment}

\author{
Bridget K. Behe ${ }^{1,4}$, R. Thomas Fernandez ${ }^{1}$, \\ Patricia T. Huddleston ${ }^{2}$, Stella Minahan ${ }^{3}$, Kristin L. Getter ${ }^{1}$, \\ Lynnell Sage ${ }^{1}$, and Allison M. Jones ${ }^{1}$
}

\begin{abstract}
ADDITIONAL INDEX WORDS. consumer research, garden center, marketing, survey,
\end{abstract} technology

SUMMARY. Eye-tracking equipment is now affordable and portable, making it a practical instrument for consumer research. Engineered to best analyze gaze on a plane (e.g., a retail shelf), both portable eye-tracking glasses and computer monitor-mounted hardware can play key roles in analyzing merchandise displays to better understand what consumers view. Researchers and practitioners can use that information to improve the sales efficacy of displays. Eye-tracking hardware was nearly exclusively used to investigate the reading process but can now be used for a broader range of study, namely in retail settings. This article presents an approach to using glasses eye tracker (GET) and light eye tracker (LET) eye-tracking hardware for applied consumer research in the field. We outline equipment use, study construction, data extraction as well as benefits and limitations of the technology collected from several pilot studies.

$\mathrm{T}$ he eyes may be the window to the soul, but they also present a window of opportunity for marketers. Davenport and Beck said it best, "The eyes don't lie. If you want to know what people are paying attention to, follow what they are looking at" (Davenport and Beck, 2001). From a marketing standpoint, the gap in correlating purchases to viewed stimuli (Pieters and Warlop, 1999; Russo and Leclerc, 1994) is closing faster than when Treistman and Gregg (1979) first documented it. Current eye-tracking hardware and software allow direct, robust eye movement measurements to assess that link between visual stimuli and purchase.

Merchandise displays are ubiquitous in retail settings, and retailers rely

\footnotetext{
The authors greatly appreciate the funding for this research provided by Michigan State University, AgBioResearch, and Project GREEEN. We also sincerely appreciate the collaboration of our garden center cooperators: Acorn and Bay Road Nurseries in Victoria, Australia, and Koetsier's and English Gardens in Michigan. The authors are also grateful for the facilitation provided by the Nursery and Garden Industry of Victoria, Australia. We also acknowledge the contributions of Tom Dudek and Lauren Tuski.

${ }^{1}$ Department of Horticulture, College of Agriculture \& Natural Resources, Michigan State University, 1066 Bogue St., East Lansing, MI 48824

${ }^{2}$ Department of Advertising, Public Relations and Retailing, College of Communication Arts \& Sciences, Michigan State University, 404 Wilson Rd., East Lansing, MI 48824

${ }^{3}$ Faculty of Business and Law, Deakin Graduate School of Business, Deakin University, 221 Burwood Highway, Victoria, Australia 3125

${ }^{4}$ Corresponding author. E-mail: behe@msu.edu.
}

on displays to be silent salespeople, draw consumers into the store, and motivate them to purchase products. Displays have the capacity to increase sales. For example, Nördfalt (2010) found that disorganized displays, which implicitly signaled cheaper merchandise, increased sales by over $900 \%$. Thus, improving our understanding of how consumers view and react to merchandise and other components of displays has both academic and practitioner relevance. The affordability and portability of eye-tracking hardware and software, along with the dearth of information about attentioncapturing stimuli in merchandise displays, make the time ideal for discovering visually captivating elements of displays that will benefit both aca-

Eye movement is not random. Visual cognitive processing requires the eyes to attend to an object, and attention requires eye movement (Russo, 1978). Eye movement is the fastest movement the human body can make (Holmqvist et al., 2011), consisting of a series of stops (fixations) and moves (saccades). Eye fixations direct attention, thus increasing mental processing demia and industry. of the meaning of an object. Characteristics about the person (top-down factors) and about the stimulus (bottom-up factors) contribute to attention, and thus, both influence the meaning derived from a stimulus. More is understood about top-down factors than bottom-up factors (Wedel and Pieters, 2008). The bottom-up factors and their role in capturing attention are only now becoming the subject of investigations due to improved affordability and compactness of eyetracking hardware.

The majority of peer-reviewed eye-tracking literature is related to reading online advertisements (Kuisma et al., 2010), television commercials (Teixeira et al., 2010) advertisements (Nixon, 1924), and package labels (Bix et al., 2009; Sorensen et al., 2012) vs. viewing merchandise displays. Wedel and Pieters (2008) call for more research on "other static visual marketing stimuli besides print ads." Investigators acknowledge that visual research on merchandise displays has not begun to develop (Chevalier, 1975; Nördfalt, 2010). For instance, "special displays are assumed to be a powerful tool to capture the customers' attention, yet this aspect is very little elaborated on in academic studies" (Nördfalt, 2010). Nördfalt postulated that learning more about capturing attention is of "great academic and practical interest."

Given the void in the literature for field research on displays and the emergence of affordable, portable eyetracking hardware and software, now is an ideal time to investigate what captures attention in displays. These devices enable researchers to get closer to the consumer in ways not possible before the advent of this technology. Anthropological studies would permit a research to follow a subject and record what she/he looked at. This technology permits not only following and recording, but a less visible method and more exact identification of what is viewed. The methods described in this paper outline the practical use of eye-tracking technology to assess merchandised horticultural products. Eyetracking technology provides a new

\begin{tabular}{llll}
\hline $\begin{array}{l}\text { Units } \\
\begin{array}{l}\text { To convert U.S. to SI, } \\
\text { multiply by }\end{array}\end{array}$ & U.S. unit & SI unit & $\begin{array}{l}\text { To convert SI to U.S., } \\
\text { multiply by }\end{array}$ \\
\hline 0.3048 & $\mathrm{ft}$ & $\mathrm{m}$ & 3.2808 \\
2.54 & inch(es) & $\mathrm{cm}$ & 0.3937
\end{tabular}

\footnotetext{
Hortednology $\cdot$ August $201323(4)$
} 
measure of bottom-up factors that literally catch consumers' attention and, hopefully, lead to an improvement of shopping experiences that result in a greater probability of purchase. This paper seeks to provide a methodology and protocol for use of two types of eye-tracking devices to aid in retail display investigations.

\section{Materials and methods Hardware}

We evaluated two types of hardware and the software used to operate the equipment: one pair of eye-tracking glasses and another eye-tracking device mounted to a computer monitor. The GET was a mobile glasses eye tracker (Fig. 1) while the LET device was the Xl Light Eye Tracker (Fig. 2) both manufactured by Tobii (Danderyd, Sweden). Both devices are well suited to indoor consumer research using displays that are away from bright or direct sunlight. Both instruments are classified as bright or light pupil, meaning they have IR (infrared) light reflected back to the device from the viewer's retina (Holmqvist et al., 2011). We summarize the comparison of 10 aspects in Table 1.

\section{Portable glasses eye tracking}

Initially, we experimented with the GET for data collection since its portability made it ideal for investigating the shopping experience in situ. The GET is a light, weighing $\approx 75 \mathrm{~g}$, but relatively rigid device that resembles safety glasses with a camera mounted near the right eye lens. To avoid damaging the glasses, they must be handled by the more-flexible earpiece parts only. Once the participant slips the GET over their head, an adjustment strap holds the GET in place. Although relatively light, the glasses do have a tendency to slip down the bridge of the nose due to the weight of the camera, so a snug fit of the strap against the head is essential for a continuously accurate recording. The GET cannot be worn over prescription glasses and with some types of contact lenses, so participants requiring them are ineligible to participate in a study.

The glasses are worn like safety glasses but wired to a $12 \times 8 \times 3-\mathrm{cm}$ recording device, which can be clipped to a belt or purse or held while shopping. The recording device operates on a battery which discharges within 30 min of use. The GET has a cable for charging the battery while the unit is still in the "on" position and should always be charging when a participant is not being recorded. Keeping the unit turned on and plugged in to keep the battery charged enables recordings to be kept in one file or within one

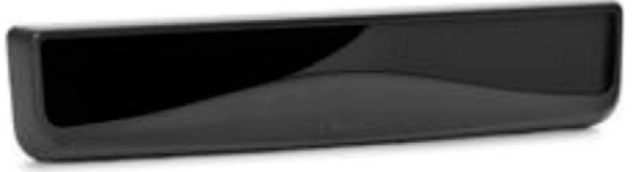

Fig. 1. Light-pupil monitor-mounted eye-tracker device [LET (model Xl; Tobii, Danderyd, Sweden)]. Image from Tobii.

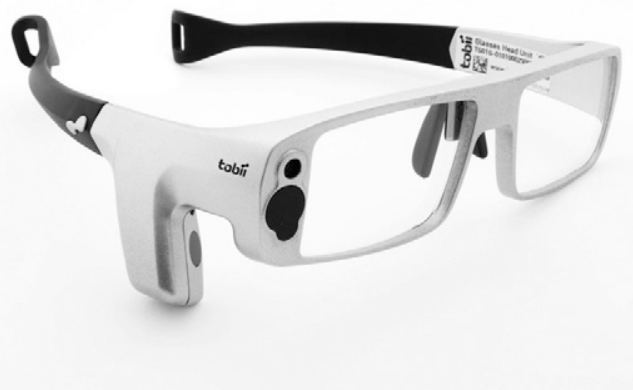

Fig. 2. Mobile glasses light-pupil eye-tracker device (GET) manufactured by Tobii (Danderyd, Sweden). Image from Tobii.

session, which is helpful at analysis time. Time management of recordings to permit frequent battery replacement is essential.

The GET device can be used independently or in conjunction with IR markers. IR markers are used in the calibration process and to aggregate visual data from one plane (e.g., a display). Consecutively numbered IR markers are included with the GET rental/purchase and are used both in the calibration process (described below) and in the displays. The glasses receive IR signals from the small $(1 \times$ $1 \times 1$ inch) IR markers and triangulate the wearer's gaze. An IR marker is held during the calibration process but otherwise installed in a display in a plane to create a "flat image" on which the visual map is created. The location of each numbered IR markers should be mapped by hand in a diagram of the display. The location of numbered IR markers can be verified with a snapshot taken with the glasses before data collection. The use of IR markers in displays also permits aggregation of all participants' visual activity in that display. The GET can be used without IR markers installed in displays, but the use of IR markers in the displays enables researchers to more efficiently aggregate data across participants.

Once the participant puts on the glasses and the recorder was turned on, the participant was calibrated. Calibration requires a flat surface near the tasks and displays. We used walls, doors, and expandable banners to calibrate participants, all with great success. We placed a mark on the floor $1 \mathrm{~m}$ from the flat surface to position participants for calibration. While a researcher held and viewed the recording device screen, she/he moved the IR marker across a flat surface as the participant stood still (without moving his/her head) and followed the marker moving only his/her eyes as the researcher moved it across the nine points needed for visual calibration. Calibration accuracy varied across the nine points by participant, but at least some readings must be made for each point to proceed with any recording. Recording of tasks or shopping commenced after successful calibration.

While calibration was relatively easy with younger participants, the process presented a consistent challenge for many study participants over age 40 years, even among those with 
Table 1. Comparison of 10 attributes for light eye tracker (LET) and glasses eye tracker (GET) devices.

\begin{tabular}{|c|c|c|}
\hline Attributes & LET $^{\mathbf{z}}$ & GET $^{\mathrm{z}}$ \\
\hline Hardware compatibility & $\begin{array}{l}\text { Users may wear some types of corrective } \\
\text { glasses and contact lenses }\end{array}$ & Users may wear some types of corrective contact lenses \\
\hline Pupils monitored (no.) & 1 & 2 \\
\hline $\begin{array}{l}\text { Light sensitivity (reducing } \\
\text { recording capability) }\end{array}$ & Moderate & High \\
\hline Data aggregation ease & Easy & Possible only through use of IR markers \\
\hline $\begin{array}{l}\text { Recording distance for } \\
\text { optimum accuracy }\end{array}$ & $1 \mathrm{~m}$ from screen & $1-15 \mathrm{~m}$ \\
\hline
\end{tabular}

${ }^{\mathrm{z}} 1 \mathrm{~cm}=0.3937$ inch, $1 \mathrm{~m}=3.2808 \mathrm{ft}$.

no corrective lenses. The lower left corner of the calibration field was an especially difficult point to calibrate since the IR sensor tracks only the right pupil and is on the right side of the GET. Without some reading at all nine points, no recording was possible. As participants' age increased, some had droopy eyelids, which interfered with calibration. Thus, we developed techniques to improve the capture of the lower left corner calibration point especially. Asking the participant say "wow" while opening their eyes as though they were surprised, was usually enough of a change to permit calibration of the lower left point. Participants with very light iris colors (blue and green), droopy eye lids, long bangs, or wearing heavy mascara had a higher probability of not being calibrated or losing eye movement recording, consistent with Holmqvist et al. (2011).

It is critical that calibration and recording be conducted with no direct or bright sunlight, since IR light from bright sunlight will overwhelm the eye-tracking sensor. We encountered this challenge during pilot studies in several garden centers, all of which had areas under partial covering, which permitted sunlight to penetrate into the displays. We found that if we conducted calibration in a dark room, it enabled us to collect data with some sunlight present after calibration. For example, we were able to capture some data when clouds passed overhead. The frequent interruption of data collection due to sunlight prompted us to explore the use of the LET. Walking participants from a calibration area to the data collection location did not appear to impact data collection or results.

The advantages of the GET are that it captures a real shopping experience while being highly portable and minimally intrusive. The device records both audio and video of a shopper's view providing a genuine glimpse into natural shopping behavior. The subject is not recorded; only what she/he views and says. The audio track can be turned off before study commencement if institutional review board (IRB) issues arose. The disadvantages of the GET are that corrective lenses may not be worn to use with the GET; older participants were challenging to calibrate; and visual data were collected only from the right eye. Another disadvantage of the GET is light sensitivity. Many garden centers have bright, direct sunlight for plant maintenance, which profoundly reduces the recording operation of the device.

STUdy DEsign with GET. Because the GET records both audio and video and tracks the participant's vision through a shopping experience, it is a powerful tool in understanding the shopping process and what is noticed and what is ignored in a retail setting. One of the challenges in designing studies with the GET is aggregating data over multiple participants. If participants are tasked to "shop as they normally would," a realistic record of their activity is made. Individual products viewed or observed can be identified and, on a frame-by-frame basis, counted to see whether the item or area of interest was viewed. Replication over several participants is challenging because individuals follow different paths in the retail space, thus making analysis cumbersome and time consuming.

One strategy used to more easily aggregate data collected with the GET was to create a set of displays with IR markers embedded in them for participants to view. IR markers set up in a plane helped to record eye activity across that plane and enabled us to identify areas of visual activity over several subjects. However, the IR markers work only when they are in the same plane, creating a flat "image" for the GET to record. This can be a challenge when display stimuli occur across different planes, such as a very deep display on step-like shelves. Adequately covering a display with IR markers in the same plane can also be a challenge. In our GET pilot studies, we used dowel rods and adhesive clay to mount the IR markers in a vertical plane. Dowels were inserted into wood blocks and blocks were distributed within a vertical plane in the display at $\approx 12$-inch intervals. The IR markers were fastened to binder clips with adhesive floral clay and attached to the dowel rods. The IR marker numbers were mapped for the display by hand and using the GET camera. Using the GET, a digital camera (or both), digital images can be taken from a point marked on the floor where the study 
participants would stand. For example, we established a mark on the floor in front a display, a point at which a digital image was taken, and asked participants to stand at this mark while viewing displays. One limitation of using the GET with IR markers is that participants may notice the IR markers as an unusual display component.

In designing studies with the GET, recording what captures attention at a very close $(<\mathrm{l} \mathrm{m})$ or very distant $(>15 \mathrm{~m})$ range results in a visual distortion called parallax. Parallax is the distortion seen in the recording and the actual position of an object when viewed along two different lines of sight. Parallax correction is set at $1 \mathrm{~m}$ by default on the GET device, but can be modified in the software depending on anticipated distance between the participant and the display to be viewed. However, distances $>15 \mathrm{~m}$ are beyond the range of correction for parallax error. As such, we recommend that when using IR markers, researchers have the participant stand at a specific point on the ground to view the display. Standing at a common mark to view the display also prevents the study participant from touching the objects on the display, to maintain display consistency.

GET TASKS AND PROTOCOL. We used the GET with IR markers to collect data at six independent retail garden centers in May 2011 and again at an independent garden center in Nov. 2011. Enlisting participants for field studies necessitated offering an incentive and actively recruiting participants (Minahan et al., 2013). We initially offered participants a $\$ 10$ gift card for the retail operation to wear the GET and complete one task three times, once for each of three displays.

Initially, the task was to identify the plant they found most attractive in each display. However, the tasks we developed evolved over time, since participants completed tasks surprisingly quickly. In general, most study participants could complete one task in less than $1 \mathrm{~min}$. We had no hypothesis about the length of time the tasks would take as no shopping processes using eye-tracking technology had been previously reported. For the initial studies, tasks were relatively simple and required only a verbal reply to keep participants' attention focused on the activity. At first, we asked study participants to select the "best" plant from a single plane display in a $1 \times 1-m$ space. We asked them to stand at a mark on the floor, view the display of high-quality, homogenous plants, and verbally respond to the task. Displays visible from one side only worked best (e.g., endcaps). Multisided or island displays, which were designed to be viewed from multiple sides, resulted in no useful data collection. This was because the IR markers could not be placed in a single plane, visible from only one side. We gradually increased the complexity of the displays in a single plane when the ease and rapidity of completing the tasks became apparent.

As we incorporated more and longer tasks (increasing the number of displays and/or the questions we asked at each display), we raised the incentive for participants from \$10 to $\$ 20$ or $\$ 25$ either in cash or as a gift card to the retailer. In all but one location, this incentive seemed sufficient to entice a participant to complete the informed consent process (required by universities), calibration, and tasks, which took 10-20 $\mathrm{min}$ in total. Having the support of retail employees, who frequently referred customers to our study, greatly helped reach our desired number of participants, dictated by a combination of statistical analyses and budget constraints. We generally recruited 25 to 60 subjects per study.

While participants conducted the remainder of their shopping, the GET captured an audio and video recording of the entire visual field as well as the areas/items at which the participant looked. While these data are not easily aggregated, viewing the shopping process produced powerfully enlightening results. For example, we were able to follow the physical path that customers followed and saw which displays captured their attention and which were ignored. Unfortunately, frame-by-frame analysis of what was viewed became too cumbersome and time consuming to yield replicable results.

\section{Light eye tracker}

Like the GET, the LET is designed to be extremely portable. This instrument, however, is magnetically mounted on a bar attached to a laptop screen or computer monitor. The manufacturer recommends using monitors $<60 \mathrm{~cm}$ (diagonal screen measure), and the monitor must have Video
Electronics Standards Association mount screw taps on the back to connect the bracket that holds the LET on the front of the monitor. The bracket expands to handle a variety of monitor widths. We found it easier to mount the bracket to the monitor first, and then plug in the power and computer cables to the LET, and finally mount the device onto the bracket. The computer connection is made through a Universal Serial Bus (USB) cable, and it is critical that this is plugged into a 2.0 USB port since it will not work with a 1.0 USB port. The monitor, computer, and LET all need a power supply, so having an appropriate number of outlets or extension cords and power strips must be planned.

Once the LET unit is powered on, the dimensions of the monitor and the angle of the bracket had to be inputted into the software at each use, either manually or from a saved file. Both a metric tape measure and electronic angle calculator are used and included to measure monitor dimensions, as well as the angle on which the LET rests on the bracket. Tobii software does not store this information but there is an option to save this to a reference file. Since every time a test is opened or reopened, the dimensions must be reentered or uploaded from the file, we recommend saving the file to the desktop for ease in locating.

The LET also needs to be calibrated to each participant's unique eye movements, similar to the calibration procedure described in the section Portable Glasses Eye Tracking. Each data collection session began with a representation of the participant's eyes in a small black box visible in the middle of the monitor. Each participant's seating was adjusted so his/her eyes were centered in the black box. The laptop operator then initiated the software calibration mode. Participants were instructed to sit comfortably still and follow the moving red and black calibration dot only with their eyes and not by moving their heads. Accuracy of data collection with the LET is much improved when head movements are minimized. The calibration process is automated with the LET, and is also a nine-point grid as previously described. However, for the LET this process is automatically queued and executed when the calibration process is initiated. The calibration 
typically took 30 to $60 \mathrm{~s}$. Participants needed to have nothing obscuring their eyes such as hair or hats. There were a few problems with heavy mascara use on some female participants with the LET. With these participants, there were several seconds of lag between completing calibration and when the software showed the researcher and study participant the calibration results. The laptop operator could then elect to do the calibration again or proceed to the test.

In addition to its small size and portability, the advantages of the LET over the GET are its ability to track both eyes and include participants who wear some types of prescription vision correction, including many types of corrective glasses. Researchers had greater difficulty in calibrating myopic subjects (who needed their glasses to read) and those wearing bifocal or trifocal lenses, especially without a line.

STUDY DESIGN WITH LET. Display images are readily incorporated into testing and are easily replicated across subjects, locations, and times of day. A large computer monitor to view static or moving images simulates a shopping experience. While not an actual shopping experience, having participants view identical images of displays made studies consistent over time and enables the manipulation of stimuli such as message content or pricing. Furthermore, products (especially plants) in photographed displays remain static over time, enhancing the reliability of the data collected.

Our studies with the LET used images of garden center displays. We digitally photographed displays with and without signs. These images were saved as .jpg files that could be manipulated with various software programs. Since the images were of different sizes and orientations (landscape vs. portrait), they were distorted (i.e., too large) when imported directly into the Tobii software. To overcome the distortion and save time adapting each image, we imported the raw images into PowerPoint (Microsoft Corp., Redmond, WA), so that the entire image could be shown on each slide, somewhat standardizing all images. In PowerPoint, we manipulated the text on the signs to make it consistent in typeface but different in content. We created two slides from the same image. Both images contained a sign with the plant name. One image displayed content information (supplemental information about ideal garden site, planting, or being a native plant), while the other image had the plant name and a separate sign that displayed the price. The software records where the eyes first fixate, which can be an artifact of the prior visual stimuli. Since we observed this phenomenon, in later studies, we added a bull's-eye on a black background to recenter their gaze. This meant that nearly all participants who looked at the bull's-eye had a similar first fixation on the next image. The slides were randomized in PowerPoint and then exported as individual .jpg files. This allowed importation of uniformly sized .jpg files into one Tobii test file rather than different sized original .jpg files from the camera images. The PowerPoint slides also served as a record of the image order, because they were numbered by PowerPoint as they were exported.

Once imported into a Tobii file, the only editing necessary within the software was how the images were advanced. Since we wanted to measure how long each participant viewed each image, we selected manual advance, as opposed to automatic advancement after a set number of seconds. Participants could use the left mouse click or any keystroke to advance the image themselves. One critical error we made was to use the "escape" key as an advance key. This proved to be an action the Tobii software could not handle, and caused the software to crash. We recommend using the left mouse click or space bar to advance the images. However, it was easier for us to have a research team member advance the slides rather than allowing the potential for error in having subjects advance the images themselves. This also created operator consistency across participants.

In addition to images, it was helpful to create several instruction slides before each task. This could be done either in PowerPoint or in the Tobii software, and we found it faster to make them in PowerPoint, since they were then ordered correctly among the images as they were imported into the Tobii software. We also discovered it was helpful to have the participant view one example to practice the protocol and to reassure them that there was no "right" or "wrong" response. The Tobii software was set up to include survey questions, but we found adding questions at the end of the slides tied up the eye-tracking computer and monitor, delaying the next participant. We also discovered that $\mathbf{3 0}$ images in total was the upper limit before fatigue symptoms emerged, which included sighing, fidgeting, and voice tone change (Miller and Jolley, 2012).

LET TASKS AND PROTOCOL. We used the LET in two garden centers in Victoria, Australia, in Mar. 2012 and two Michigan garden centers in May 2012. Wanting to simulate the shopping process as realistically as possible, we asked study participants to view images of garden center displays on the computer screen and respond to questions that pertained to the displays. To conduct the studies, we took the equipment to the retail site to recruit participants who were already primed to view plants and gardeningrelated displays (Minahan et al., 2013). We used a folding table with three chairs; one each for the computer operator, data collector, and participant. The table required sufficient space to accommodate the monitor and laptop unit and room for participants to sign the informed consent form.

Once the IRB approved consent form was signed, we asked the participant to sit comfortably still. The Tobii software assigned each new participant the next consecutive number as a participant identification number. This number was also recorded by the data recorder on the data collection sheet. Next the laptop operator verbally told the participant that he or she would see a series of displays.

The participant simply sat comfortably, viewed the monitor as the display images were shown, and verbally responded to the questions. As the participant viewed each display, he/she was instructed to verbally rate each display with a 10-point Likert scale ( $1=$ not at all likely to purchase, 10 = very likely to purchase) using whole numbers, as some individuals want to use half units. The scale itself appeared to be straightforward, but participants sometimes had questions, often after the third or fourth display, about what "would likely to buy" meant. The scale used for some slides of the study was attractiveness, and it did not raise any questions. We advanced the study to the next image immediately after the verbal response was given. This permitted measurement 
of the length of time each image was viewed until a decision was made. We added a "thank you, you are finished" as a final instruction image at the end of the study to end on a nontest image. The escape keystroke completed the test and saved the recording, whereas other keystrokes would not advance or end the recording. Having a research team member enter data kept participants from handling the computer, which eliminated diverting their attention from the display and creating possible errors in data entry or malfunctions by pressing the wrong keys.

Our studies included some of the following questions or statements: 1) How visually appealing do you find this display? 2) How likely would you be to buy something from this display? 3) How effective do you find this display? 4) I enjoy looking at this display. 5) I can picture myself buying one of the items from this display. 6 ) I would recommend an item in the display to a friend. 7) The items in this display are not worth my money.

Because we were unsure as to the length of time it would take to calibrate a participant and have them complete a task, we initially only collected gender by visual observation and verbally asked for postal code. Calibration could take from 1 to $5 \mathrm{~min}$ or more; the tasks were completed in less time than calibration. We soon discovered that the test was completed rapidly $(<\mathrm{l}$ min) by most participants, so we could also easily collect additional demographic information and ask purchase behavioral questions without fatiguing the study participants. For subsequent studies, we collected supplemental information separately using a paper-and-pencil survey form.

\section{Data handling}

Metrics. Before data analysis began, areas of interest (AOIs) for each image were identified (Fig. 3). AOIs can be created using the software either a priori or post hoc as geometric or free form shapes around products, items or other portions of the image. In our studies, AOIs were drawn around signs and plants. The Tobii software produces a tremendous number of metrics that can be used in data analysis. After working with several data sets, we selected three key metrics to use in subsequent AOI analysis: time to first fixation (TTFF), first fixation duration (FFD), and total visit duration (TVD). Fixations are the stops in saccades. These fixations can be counted (FC) on the image as a whole or by using AOIs within an image. We interpreted the time to first fixation, measured in milliseconds, as the time it took for a participant to first "see" an AOI, and the first fixation duration as the ability of that first area or item to hold attention. FC revealed how often a participant viewed that AOI or refocused attention to that element. Visits are fixations on an AOI. Visit duration is equal to the fixation count multiplied by the fixation duration, yielding a measure of time spent attending to a particular $\mathrm{AOI}$, and we interpreted this measure as one of cognitive processing (thought) through attention.

It is important to note that some participants view AOIs while others do not, producing a zero FC and zero TVD. We opted to include this zero FC and zero TVD and also captured the percent of consumers who did not view an AOI. In one pilot study, we found that less than $1 \%$ of the participants, on average, did not view the item identified as a distinct AOI while 15\% or more failed to view the price AOI in the same image (P.T. Huddleston, B.K. Behe, S.M. Minahan, and R.T. Fernandez, unpublished data). The lack of FC on an AOI is an important consideration before data export because the metrics can be exported either including or excluding those zero counts. We found the zero counts useful as supplemental metrics. Once the useful metrics for analysis were identified, the data were easily exported to a text file that could then be converted to any file type the user desires.

INDIVIDUAL RESPONSE DATA. With both GET and LET, recordings are captured into one file per participant, sequentially labeled in the order in which they were created. At this point, several alternatives for data analysis are open to the researcher. Each participant has a recording, which can be played and viewed individually. The GET shows the progress of the participant through the tasks with both audio and video output. The LET can also show a recording of the progression of the participant through the images.

Because we collected supplemental information (e.g., demographics, past purchases, attitudes, etc.) on a paper survey for some of our studies, these data were merged with visual data after the visual data were extracted from the Tobii software. This was most easily done using an Excel (Microsoft Corp.) spreadsheet.

SCAN PATHS. Both GET and LET recordings show the scan path or the progression of eye movement through the scenes. A scan path is the progression of eye movement through a display or image, created for one

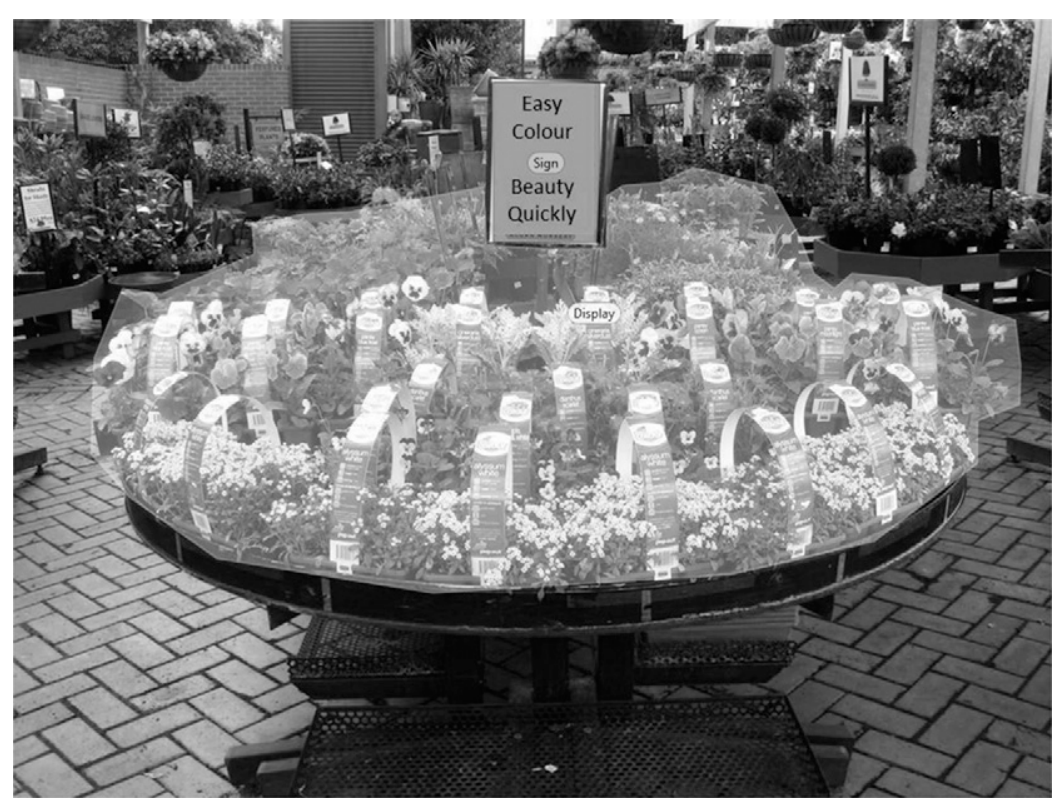

Fig. 3. Actual test image showing two areas of interest added for analysis. Areas of interest were drawn on the sign and plant material (merchandise). 
participant at a time. The diagram of a scan path can be exported as a static image (Fig. 4) or as a movie. The scan path shows a series of numbered geometric shapes (default is a circle), which indicate the order and point at which the participant looked at a particular portion of the image or display. Larger shapes indicate longer viewing time.

Heat Maps. One method to view aggregated data overall recordings or selected recordings is with heat maps. Maps with "hot spots" can be generated by the software, and areas of visual saturation are denoted using a color gradient with a dark red color indicating greater viewing transitioning to green indicating lesser interest. Areas capturing no visual attention have no color superimposed over them. The heat map images can be exported as movies or .jpg files, and we found it helpful to export one image without the heat map to have as a comparison with the image with the heat map (Fig. 5).

\section{Results}

The Tobii software is capable of calculating a wide range of metrics with regard to a stimuli's visual interest. Some analyses (i.e., mean time to view image or AOI) can be conducted within the Tobii software, but the license is bound to one computer, and it is not possible for multiple users to perform simultaneous analyses on separate computers. We recommend exporting the desired metrics and integrating any supplemental data (such as verbal ratings or written survey data) outside the proprietary software to Excel or another data analysis software package. This integration is not trivial but will persist well beyond the site license for the software.

Our experience demonstrates that a thorough familiarity with eyetracking protocols and equipment is essential for successful data collection because of the complexity of the technology and its sensitivity to environmental conditions (i.e., sunlight). This technology is a highly useful tool in the consumer research arena. Since the equipment is portable, data collection can occur in situ where consumers can be recruited during their normal shopping trip rather than in a laboratory outside a normal shopping experience. Both the GET and LET accurately and precisely track what consumers are

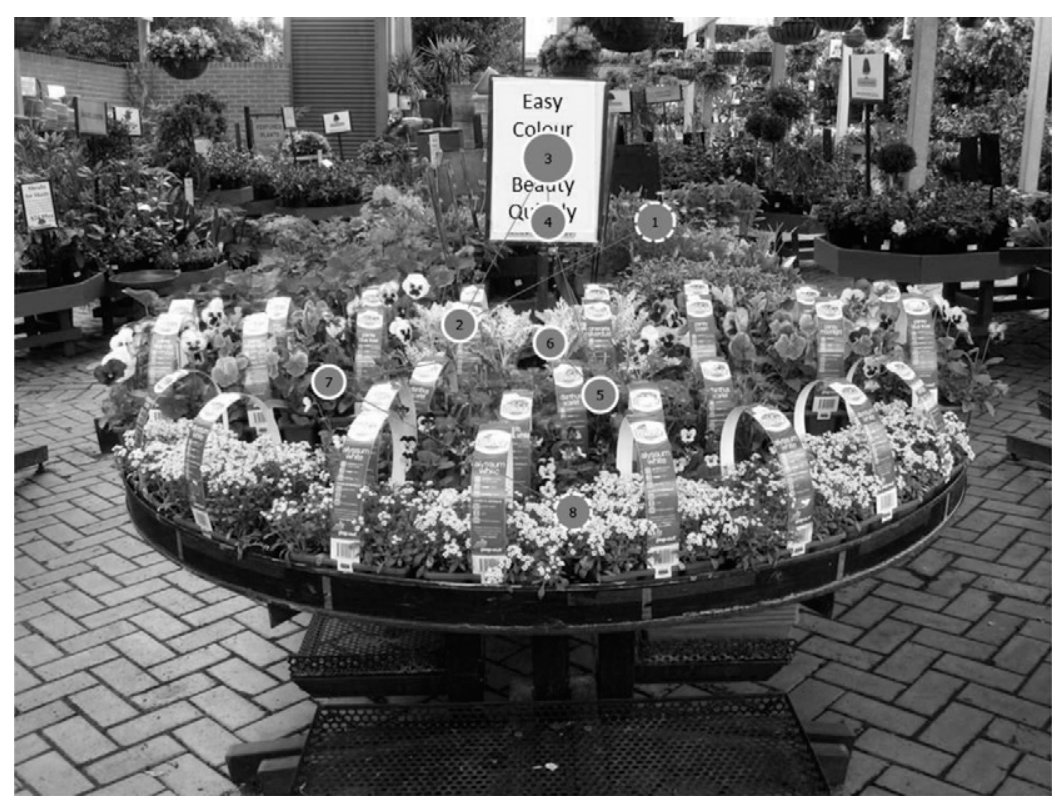

Fig. 4. Actual test image showing scan path depicting the visual progression through the image for one participant. Circle diameter is an indication of relative visit duration on that point.

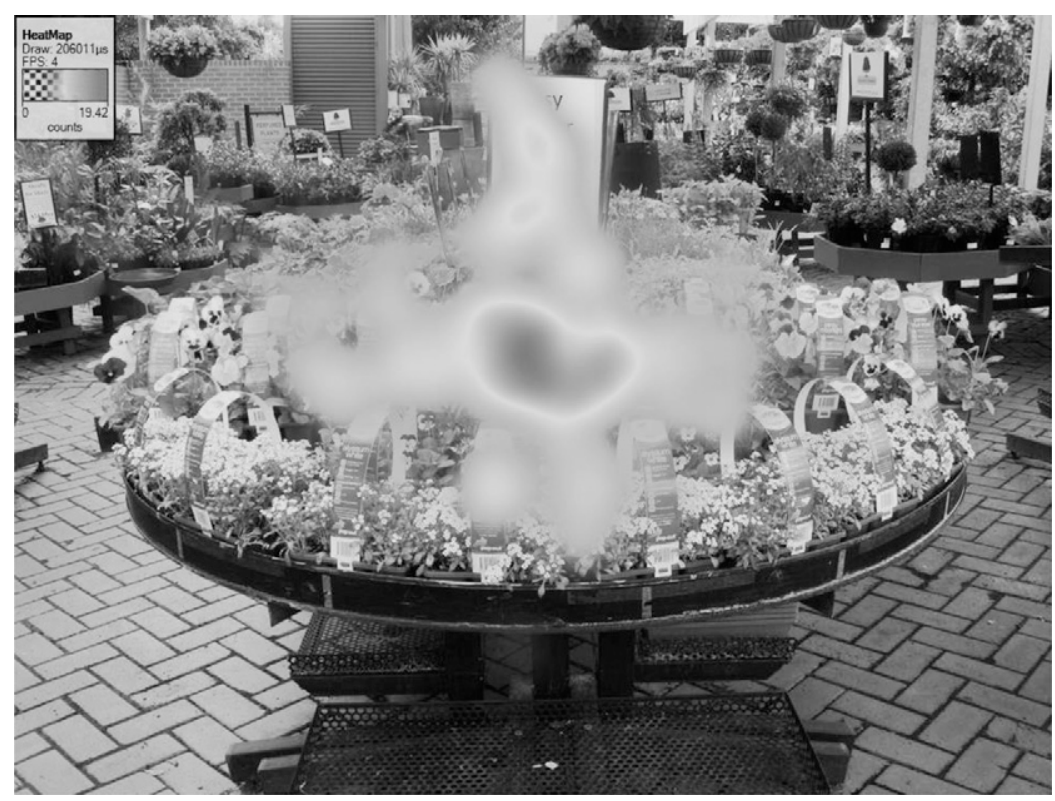

Fig. 5. Actual test image with visual heat map superimposed to show aggregation of visual data over several participants. Darker areas on the image indicate more visual activity; transparent areas show less intense visual activity.

viewing. Several measures to track eye movement and indirectly measure attention are produced. The hardware can substantially reduce, if not eliminate, researcher bias and provide instant recording of data, which eliminates transcription errors. The technology allows for time, sequence, and frequency data, which can all be combined with supplemental attitudinal, behavioral, or demographic data for analysis.

Despite the advantages of eyetracking technology in capturing accurate data regarding consumer viewed displays, there are several drawbacks to this technology. First, the inability to wear prescription glasses with the GET was a great limiting factor when the retail demographic is 
of an age where vision correction is common, as it was for our investigations. Recruiting subjects who do not wear corrective lenses is an option, but may bias the sample. Second, once the site license expires, results become unavailable and data export is no longer possible. It is advisable to download all possible metrics before site license expiration. Additionally, this particular manufacturer ties the data collection and analysis to one computer per license, substantially limiting the number of persons who can collect and analyze data at any given time. Purchasing multiple licenses would avoid this issue. Third, since the GET and LET devices are not "plug and play", on-site formal training provided by the manufacturer is beneficial for smooth operation of the eye-tracking equipment. However, there is a fee for on-site training. Experience with the technology is also advantageous, as there are small glitches or little bugs in the system that can occur. However, this report is based on one manufacturer's system; other brands might operate differently and exhibit different types of advantages and disadvantages although the principles will be similar.

Our immediate focus was limited to horticulture retail display settings, which rely heavily on fixtures exposed to some sunlight to permit plant growth. Neither the GET nor LET can be consistently used in retail venues where ambient sunlight interferes with eye-tracking equipment. Thus, the eyetracking technology may perform differently in other retail settings, such as grocery stores. In particular, the GET may be better suited to indoor settings that are unaffected by sunlight. Another limiting factor was that we experienced other customers purchasing products from test displays over the course of our studies using the GET, changing what successive study participants could view. Additionally, the IR markers distracted some study participants and we often had some visual activity on the markers themselves.
Display consistency became an advantage of the LET as it produces more replicable results, since participants viewed identical display images.

GET and LET eye-tracking hardware are practical instruments for the investigation of consumers' attention to display stimuli. Advancements in eye-tracking technology have moved investigations of attention capturing elements beyond the act of reading information to knowledge of which display elements captivate consumers. These uses should be helpful in understanding bottom-up factors that contribute to the effectiveness of merchandise displays. Future studies should include more diverse retail settings to test the robustness of eyetracking data collection methods. Another potential avenue for exploration is the manipulation of merchandise placement in addition to manipulation of signage information. These studies will undoubtedly add to the body of knowledge and expand retail theory, as well as contribute to the practical goal of increasing sales on the retail sales floor based on proven understanding of what display elements capture consumer attention and motivate purchase.

\section{Literature cited}

Bix, L., N.M. Bello, R. Auras, J. Ranger, and M.K. Lapinski. 2009. Examining the conspicuousness and prominence of two required warnings on OTC pain relievers. Proc. Natl. Acad. Sci. USA 106(16): 6550-6555.

Chevalier, M. 1975. Increase in sales due to in-store display. J. Mktg. Res. 12(4): 426-431.

Davenport, T.H. and J.C. Beck. 2001. The attention economy: Understanding the new currency of business. Harvard Business School Press, Cambridge, MA.

Holmqvist, K., M. Nystrom, R. Andersson, R. Dewhurst, H. Jarodzka, and J. Van der Weijer. 2011. Eye-tracking: A comprehensive guide to methods and measures. Oxford Univ. Press, New York City, NY.
Kuisma, J., J. Simola, L. Uusitalo, and A. Oorni. 2010. The effects of animation and format on the perception and memory of online advertising. J. Interactive Mktg. 24(4):269-282.

Miller, M.L. and J.M. Jolley. 2012. Consumer research design explained. 8th ed. Cengage Learning, Independence, KY.

Minahan, S.M., P. Huddleston, B. Behe, and R.T. Fernandez. 2013. Conducting field research in retail stores: A meandering path to a successful research project. Intl. Rev. Retail Distrib. Consum. Res. 23(2):189-203.

Nixon, H.K. 1924. Attention and interest in advertising. Arch. Psychol. 72:5-67.

Nördfalt, J. 2010. Improving the attentioncapturing ability of special displays with the combination effect and the design effect. J. Retailing Consum. Serv. 18(3): 169-173.

Pieters, R. and L. Walrup. 1999. Visual attention during brand choice: The impact of time pressure and task motivation. Intl. J. Res. Mktg. 16(1):1-17.

Russo, J.E. 1978. Eye fixations can save the world: A critical evaluation and a comparison between eye fixations and other information processing methodologies. Adv. Consum. Res. 5:561-570.

Russo, J.E. and F. Leclerc. 1994. An eye fixation analysis of choice processes for consumer nondurables. J. Consum. Res. 21(2):274-290.

Sorensen, H.S., J. Clement, and G. Gabrielsen. 2012. Food labels: An exploratory study into label information and what consumers see and understand. Intl. Rev. Retail Distrib. Consum. Res. 22(1): 101-114.

Teixeira, T.S., M. Wedel, and R. Pieters. 2010. Moment-to-moment optimal branding in TV commercials: Preventing avoidance by pulsing. Mktg. Sci. 29(5): 783-804.

Treistman, J. and J.P. Gregg. 1979. Visual, verbal, and sales responses to print ads. J. Advert. Res. 19(4):41-47.

Wedel, M. and R. Pieters. 2008. A review of eye-tracking research in marketing. Rev. Mktg. Res. 4(5):123-147. 